\title{
Biological Roles of Protein-Coding Tandem Repeats in the Yeast Candida Albicans
}

\author{
Matt Wilkins ${ }^{1}$, Ningxin Zhang ${ }^{2}$ and Jan Schmid ${ }^{2, *}$ (i) \\ 1 Centre for Professional and Continuing Education, Massey University, \\ Palmerston North 4412, New Zealand; M.C.Wilkins@massey.ac.nz \\ 2 Institute of Fundamental Sciences, Massey University, Palmerston North 4412, New Zealand; \\ N.X.Zhang@massey.ac.nz \\ * Correspondence: j.schmid@massey.ac.nz; Tel.: +64-6-951-7717
}

Received: 23 May 2018; Accepted: 27 June 2018; Published: 29 June 2018

\begin{abstract}
Tandem repeat (TR) DNA mutates faster than other DNA by insertion and deletion of repeats. Large parts of eukaryotic proteomes are encoded by ORFs containing protein-coding TRs (TR-ORFs, pcTRs) with largely unknown biological consequences. We explored these in the yeast Candida albicans, an opportunistic human pathogen. We found that almost half of $C$. albicans' proteins are encoded by TR-ORFs. pcTR frequency differed only moderately between different gene (GO) categories. Bioinformatic predictions of genome-wide mutation rates and clade-specific differences in pcTR allele frequencies indicated that pcTRs (i) significantly increase the genome-wide mutation rate; (ii) significantly impact on fitness and (iii) allow the evolution of selectively advantageous clade-specific protein variants. Synonymous mutations reduced the repetitiveness of many amino acid repeat-encoding pcTRs. A survey, in 58 strains, revealed that in some pcTR regions in which repetitiveness was not significantly diminished by synonymous mutations the habitat predicted which alleles were present, suggesting roles of pcTR mutation in short-term adaptation and pathogenesis. In C. albicans pcTR mutation apparently is an important mechanism for mutational advance and possibly also rapid adaptation, with synonymous mutations providing a mechanism for adjusting mutation rates of individual pcTRs. Analyses of Arabidopsis and human pcTRs showed that the latter also occurs in other eukaryotes.
\end{abstract}

Keywords: protein coding DNA tandem repeats; mutation rate; proteome evolution; amino acid tandem repeats; Candida albicans; synonymous mutations; Red Queen's race; contingency genes

\section{Introduction}

Tandem DNA repeats (TRs) are common in protein-coding regions of eukaryotic genomes [1]. The length of the repeated unit varies greatly, from a single base to hundreds of bases (units of $<10$ bases are commonly referred as microsatellites, longer units as minisatellites) [1]. TR regions are believed to arise by insertions of transposable elements or by replication slippage. Under the latter scenario, TR regions arise in an area between two non-contiguous copies of the same short sequence: the transient disassociation of DNA polymerase after replicating one copy and its re-joining at the other copy leads to a duplication of the region in between. Subsequently the number of copies of the duplicated region increases further by replication slippage in the duplicated region, or by homologous recombination [1-4].

Once established, TR regions mutate orders of magnitude faster than nonrepetitive DNA by insertion and deletion (indels) of repeat units, caused also by replication slippage and homologous recombination [5]. It is well established that indels of repeat units can have selectable phenotypic effects; examples include heritable disease in humans, changes in adhesion, alterations of regulatory 
networks and evasion of immune responses by pathogens [1,5-8]. For repeat regions that encode parts of proteins the alterations that cause such effects are by no means restricted to indel-induced frameshifts. Many protein-coding TRs (pcTRs) encode amino acid tandem repeats and indels will increase or decrease the length of these amino acid repeat regions. The effect on protein function may often be too subtle to be directly demonstrable $[9,10]$ but may still lead to selectable fitness differences, in particular in microbial species. Because of their large effective population sizes, in microbial species even mutations with minute impact on fitness are under selection [11-13]. It is not uncommon for such series of encoded amino acid motifs to be conserved between species [14-17], an indication that they can confer selective advantages. In addition, a given habitat will often contain large numbers of microbial cells and this population will contain a pool of pcTR region-containing ORF (TR-ORF) variants, continually replenished by pcTR mutation. This provides an opportunity to use TR mutation for rapid adaptation if the habitat changes or to move to other habitats; that is, TR-ORFs can function as contingency genes $[8,18]$.

The yeast Candida albicans is a frequent commensal colonizer of humans, mainly their gastrointestinal tract, and humans are apparently its primary habitat $[19,20]$. C. albicans can also cause both superficial and life-threatening systemic disease [19]. Its genome contains large numbers of pcTRs [21] and it is possible that pcTR mutation-generated protein variants could play a role in the transition from commensal to pathogen [16]. It is thus of interest to establish the biological role of pcTRs in this species and if such roles include roles in short-term adaptation.

C. albicans has two features that also recommend it as a simple model for investigating the evolutionary role of pcTRs in general. One is that its mode of reproduction is almost exclusively clonal [22]. Thus TR-ORF allele combinations in the genome of a given strain are unlikely to result from a recent sexual recombination event but are likely to have coevolved. The second is the small number of ORFs containing introns [23]; as a result, disruption by introns should only have a minimal impact on the evolutionary biology of amino acid repeat-encoding TR regions.

These reasons led us to undertake a genome wide survey of $C$. albicans TR-ORFs with the aim of elucidating the evolutionary consequences and role of pcTR mutation.

\section{Materials and Methods}

\subsection{Analysis of Tandem Repeats in the C. albicans Genome}

These analyses were based on FASTA files of coding sequences (CDS) and genomic gene sequences (including untranslated regions and introns), downloaded from the Candida Genome Database [24] (http:/ / www.candidagenome.org; SC5314 assembly 22; accessed in April 2018).

For the analysis of TRs we wrote software (TRANALYSER) based on the SERV package designed by Legendre et al. [25]. SERV finds tandem repeat stretches using Tandem Repeats Finder [26] and for each stretch calculates a so-called VARscore. VARscores (i) predict the variability of a TR stretch, that is, the probability that when different individuals are assessed, their stretches will differ in length as a result of indels and (ii) provide an estimate of the frequency at which indels occur, that is, the rate of mutation (by insertions and deletions) of a TR-region [25].

Tandem Repeats Finder often reports multiple overlapping TR stretches for a given region of DNA, with longer stretches often corresponding to multiples of shorter stretches [26]. TRANALYZER combines such overlapping stretches into TR regions and uses a weighted sum of VARscores of TR stretches to calculate a VARscore for the entire TR region (REGIONscore). To do so, it first identifies the TR stretch with the highest VARscore (or several if they do not overlap) and then adds to it the VARscores of non-overlapping parts of other stretches with lower VARscores (Figure 1a). Because the non-overlapping parts of the other stretches may in themselves not represent a TR, the VARscore added is calculated as a fraction of the entire VARscore of these stretches, based on the degree of non-overlap.

Mutation rate is exponentially related to VARscore and simply adding VARscores and fractions of VARscores to each other would therefore produce scores that suggest too high a differential between 
the mutation rate of a region and the mutation rate of its highest-scoring stretch. Therefore, before summing, VARscores are converted to mutation rate estimates (indels per generation; ipg) using the relationship between the two parameters experimentally determined in Saccharomyces cerevisiae [25]:

$$
i p g=5 \times 10^{-7} \times \mathrm{e}^{1.4 \times \text { VARscore }}
$$

After the mutation rates have been added, $i p g^{-1}$ can be applied to back-convert the sum back onto a VARscore for the entire region, that is, the REGIONscore.

Thus, the REGIONscore can be defined as follows. For a region of length $n$, consisting of $k$ stretches with individual VARscores $v_{i}$ and length $l_{i}, i=1, \ldots, k$, the REGIONscore is:

$$
\text { REGIONscore }=i p g^{-1}\left(\sum_{i=1}^{n} \frac{i p g\left(v_{m}\right)}{l_{m}}\right),
$$

where $v_{m}$ is the maximum VARscore over those stretches defined at the $i$ th base pair and $l_{m}$ is the corresponding length of that stretch.

To estimate the maximum possible REGIONscore of a TR region encoding a given amino acid sequence, TRANALYZER generates permutations of the region in two ways (Figure $1 \mathrm{~b}$ ). The first is to use, for each amino acid, only one of the codons that is used, in the original TR-region, to encode that amino acid (one codon permutation). For instance, if in a TR region serine is encoded by AGT and AGC and proline in the region is encoded by CCT and CCC, then TRANALYZER generates four alternate TR regions in which only the codons AGT and CCT or only AGT and CCC or only AGC and CCT or only AGC and CCC are used. If there are more than 500 possible one codon permutations, then 500 are randomly picked for further analysis. The second approach (random permutation) involves switching the position of the existing codons in the sequence without altering the amino acid sequence encoded (or the number of times each of the codons is used; 100 such random permutations are generated). Note that when a TR region does not begin at the first base of a codon or does not end at the last base of a codon, these partial codon sequences are not altered.

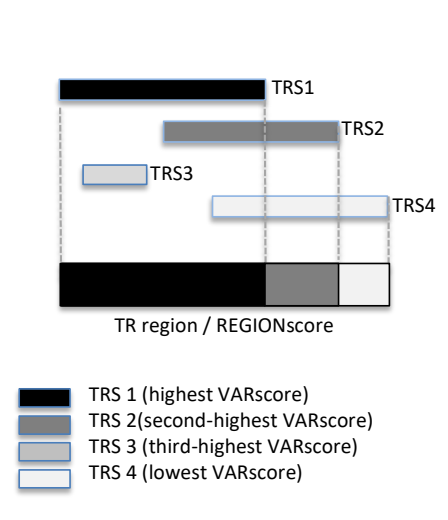

(a)

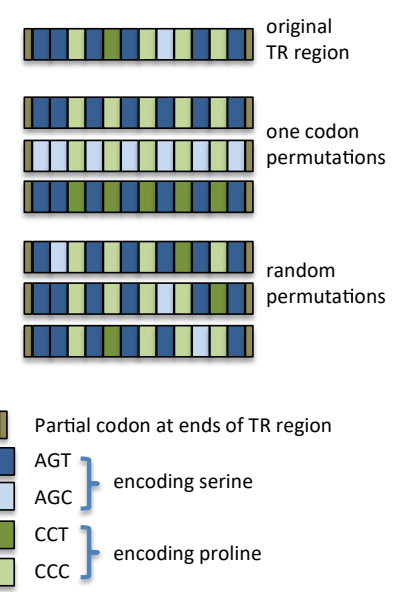

(b)

Figure 1. TRANALYZER processing of TR sequences (a) Determination of REGIONscores. REGIONscores are calculated by adding, to the VARscore of TR stretches (TRS) with higher VARscores, fractions of VARscores of lower-scoring stretches. The fraction of the score of the lower-scoring stretch added corresponds to the fraction of non-overlap with the higher scoring stretch. If an entire lower-scoring stretch is in a region covered by a higher-scoring stretch (such as TRS3), its VARscore is not considered; (b) Search for the maximum REGIONscore of a TR region encoding a series of serine/proline repeats by permutating it, without altering the amino acid sequence, by using either only one type of codon for each amino acid or by randomly shuffling codons. 
Tandem Repeats Finder is then used to find repeat stretches in each permutated TR region and a REGIONscore is calculated. Note that the repeat stretches in the permutated TR regions may not necessarily always overlap. If so, the REGIONscore is the sum of the scores of non-overlapping TR regions and/or TR stretches in the permutated TR region. The highest REGIONscore amongst all permutations and the original REGIONscore is considered to be the maximum possible REGIONscore.

TRANALYSER is an open source software. The code can be accessed at https://mattwilkins@ bitbucket.org/mattwilkins/candida.git.

The above analyses were carried out on TR regions detected in CDS and introns could interrupt $\mathrm{TR}$ regions in the genomic sequence. We therefore identified intron-containing genes by comparing sequence lengths of CDS and genomic DNA for each ORF retrieved from the C. albicans genome database. In C. albicans introns are rare and apparently almost never interrupt TR-regions (see Results). We therefore included intron-containing genes in all further analyses of $C$. albicans TRs.

\subsection{Analysis of Tandem Repeats in Other Genomes}

We used TRANALYSER to also analyse protein-coding sequences in the genomes of Arabidopsis thaliana and in the human genome. Because these genomes are large and these analyses are computationally intensive, we restricted them to a selection of genes.

For A. thaliana all representative gene model sequences were downloaded in April 2018 from The Arabidopsis Information Resource (TAIR) at https:/ / www.arabidopsis.org/download/index-auto.jsp? dir=\%2Fdownload_files\%2FSequences\%2FTAIR10_blastsets(TAIR10_cds_20110103_representative_ gene_model_updated;TAIR10_seq_20110103_representative_gene_model_updated).

For the human genome, a random selection of 8394 CDS and ORF sequences were downloaded from the UCSC Genome Browser in May 2018 (http:/ / genome.ucsc.edu/cgi-bin/hgTables). To avoid low quality gene models, only genes that encoded proteins with an entry at the high quality swiss-prot database (https:/ / web.expasy.org/docs/swiss-prot_guideline.html) were considered for selection.

The genomes of both species contain large numbers of introns. Therefore, we identified TR regions both in the genomic sequence and in the CDS of each gene. We determined for each TR region in the genomic sequence if it was identical to, or contained, a TR region present in the CDS. If so, we considered the TR region to be a pcTR region, uninterrupted by introns. Based on these analyses we calculated the total number of TR-regions in genes and the percentage of such regions that is not interrupted by introns.

For TR regions that did not overlap with introns we calculated REGIONscore and determined the maximum possible REGIONscore. In the case of Arabidiopsis the latter was done only for $630 \mathrm{TR}$ regions in 502 TR-ORFs, selected from amongst all 9129 regions in 7115 genes. Selection was based on the ranking of REGIONscores so that the set was equally representative across the entire range of REGIONscores.

\subsection{Determination of Allele Distributions for Six TR Regions}

To determine TR region length allele distributions, 58 strains (Table S1), were chosen [27] to best represent the genotypes of an international collection of 461 DNA fingerprinted commensal and disease-causing isolates [28-33] All of these strains had only been briefly cultured after their isolation and had subsequently been maintained as glycerol stocks at $-80^{\circ} \mathrm{C}$.

TR regions in genes IFF6, HYR3, PGA55, EAP1 and orf 19.1075 were amplified by colony PCR [17]. To do so, a portion of a C. albicans colony from each strain was picked with a $10 \mu \mathrm{L}$ pipette tip and mixed with $20 \mu \mathrm{L}$ PCR reaction mix. The mix contained the pair of primers that amplified a region (Table S2), 1 U Taq DNA polymerase (Qiagen Pty Ltd., Clifton Hill Vic, Australia), $4 \mu \mathrm{L}$ of Q-buffer, $2 \mu \mathrm{L}$ of $10 \times$ PCR buffer supplied by the manufacturer (Qiagen), 10 pmol of each primer and $200 \mu \mathrm{M}$ of each dNTP (Roche Diagnostics, Auckland, New Zealand). The cycling conditions, varied according to primer sets and the size of the products and included an initial incubation for 5 min at $96{ }^{\circ} \mathrm{C}$, followed by 30 cycles of $45 \mathrm{~s}$ at $94{ }^{\circ} \mathrm{C}, 45 \mathrm{~s}$ at $50-60{ }^{\circ} \mathrm{C}$ and $30 \mathrm{~s}$ to $3 \mathrm{~min}$ at $72{ }^{\circ} \mathrm{C}$. All PCR protocols 
included a final $5 \mathrm{~min}$ extension step at $72{ }^{\circ} \mathrm{C}$. Reactions were carried out in an Eppendorf Mastercycler thermocycler (Eppendorf, Hamburg, Germany). As a positive control, the products were also amplified in laboratory strain SC5314.

The sizes of PCR products were measured on $2 \%$ agarose gels, against a $1 \mathrm{~kb}$ plus DNA ladder (Invitrogen Life Science Technologies, Auckland, New Zealand). Sizes of select PCR products were also determined on a 3730 Genetic Analyzer (Applied Biosystems, Waltham, MA, USA) [16] to verify visually detected size differences on gels (and that bands apparently of the same size indeed represented product of identical length).

\section{Results and Discussion}

\subsection{Almost Half of the ORFs in the C. albicans Genome Are TR-ORFs}

The Candida albicans diploid genome assembly (strain SC5314, assembly 22) contains 12421 different ORFs (representing 6226 loci). We used Tandem Repeats Finder [26] to identify TR stretches in the 12421 coding sequences (CDS) from these ORFs. We identified 5708 TR-ORFs (i.e., ORFs containing one or more TR stretches) corresponding to 2936 gene loci (on some occasions TR stretches were detected only in one copy of a gene; Table S3). Tandem Repeats Finder often identifies multiple partially or wholly overlapping repeat stretches in the same region of DNA. We therefore wrote software, part of the TRANALYSER package we developed for the analyses of TR-ORFs reported here, that combined any overlapping repeats into TR regions (see Table 1 for some examples of TR regions and Table S3 for all TR regions). The 5708 TR-ORFS contained 12122 such TR regions. Introns are present in 252 TR-ORFs and these could interrupt some TR regions we had discovered in the CDS.' However, of 31 TR regions in intron-containing TR-ORFs analysed, none was disrupted by an intron and thus such interruptions seem rare. Thus $46 \%$ of all ORFs in the C. albicans genome are TR-ORFs.

Over half of TR-ORFS ( 2988 of 5709 TR-ORFs) contained only one TR region but up to 21 could be present in a TR-ORF (Table S3). TR region sizes varied from $20 \mathrm{bp}$ to $5156 \mathrm{bp}$ (median: $35 \mathrm{bp}$; average $48 \pm 105 \mathrm{bp}$ ). The repeated amino acid motifs encoded by the repeat units ranged in length from 1 amino acid to 162 amino acids (see Table 1 for some examples of TR regions and Table S3 for all TR region-encoded amino acid regions). The combined length of all TR-regions was $590 \mathrm{~kb}$, equivalent to $3 \%$ of all $18 \mathrm{Mb}$ protein-coding DNA.

Table 1. Examples of TR region.

\begin{tabular}{|c|c|c|c|}
\hline TR Region ${ }^{1}$ & $\begin{array}{l}\text { REGION } \\
\text { Score }\end{array}$ & $\begin{array}{c}\text { TR Unit } \\
\text { Lengths }(b p)^{2}\end{array}$ & Nucleic Acid and Amino Acid Sequence of Regions ${ }^{3}$ \\
\hline \multirow[t]{2}{*}{$\begin{array}{c}\text { C2_10280C_B } 3 \\
\text { (SPT23) }\end{array}$} & 1.01 & 3 & $\begin{array}{l}\text { ACAACAACAACAACAACAACAACAACAACAACA } \\
\text { ACAACAGCAGCAGCAACAGCAACAACAGCAA }\end{array}$ \\
\hline & & & QQQQQQQQQQQQQQQQQQQQQ \\
\hline \multirow[t]{2}{*}{$\begin{array}{c}\text { C1_01560W_B } 1 \\
(\text { SIZ1) }\end{array}$} & 0.92 & $6,3,12,24$ & $\begin{array}{c}\text { ACAGCAACAGCAACAGCGACAACTTCGACA } \\
\text { ACTAGAACAGCAGCAACGGCTACAGCGAC } \\
\text { AGCAATGGCAACAGCAACAGCAACAACT } \\
\text { ACAGCAACAACAACAACAACTTCCTCGC } \\
\text { CAACTTCCCCAACAGTCTCCC } \\
\text { CGACAACTTCCCCAACAG }\end{array}$ \\
\hline & & & $\begin{array}{c}\text { QQQQQRQLRQLEQQQRLQRQQWQQQQ } \\
\text { QQLQQQQQQLPRQLPQQSPRQLPQQ }\end{array}$ \\
\hline \multirow[t]{2}{*}{ C2_02330W_B 1} & 0.28 & 4 & САСТТАСТСАСТСАСТСАСТСАСТСАСТС \\
\hline & & & LTHSLTHSL \\
\hline C1_09690W_B 2 & -1.15 & 12 & TTTCTTGCCAGATTTCTTACCAGA \\
\hline (MLS1) & & & FLPDFLP \\
\hline
\end{tabular}

${ }^{1}$ Name of ORF in the Candida genome database followed by the number of the TR region. For ORFs of named genes this is followed by the name of the gene, in brackets. ${ }^{2}$ Lengths of repeat units as reported by TR finder. ${ }^{3}$ The amino acid sequences do not include a translation of partial codons at the beginning of end of a TR region. 


\subsection{TR-ORFs Considerably Increase the Genome-Wide Frequency of Mutations in C. albicans Exons}

The mutation rate of a TR region depends on a number of characteristics, mainly the length of the repeat unit, the number of units present and their purity (the sequence similarity of the repeating units) [25]. Legendre et al. [25] developed a software (SERV) that generates a score (VARscore) for a TR region, based mainly on these characteristics. VARscores indicate how likely it is that allelic variants of a repeat region will be found when populations are assessed. While VARscores are not direct predictors of mutation rate, these authors established, in haploid S. cerevisiae, that mutation rate is exponentially related to VARscore [25].

SERV calculates VARscores for TR stretches which often overlap, and we combined these to calculate a REGIONscore, a VARscore for a TR region composed of a set of overlapping stretches (see Methods for details and Table 1 for examples of TR regions and their REGIONscores).

The average REGIONscore of all pcTR regions was -0.449 (range -1.833 to 3.917 ) corresponding to an estimated rate of $2.7 \times 10^{-7}$ mutations per region per generation, or $3 \times 10^{-3}$ mutations per generation in one of the 12,221 pcTR regions in the genome, according to the relationship between VARscore and mutation rate established by Legendre et al. [25]. We had earlier measured pcTR mutation rates in C. albicans $[16,17]$ for two genes (PNG2 and SSR1). In both cases these were $\sim 7$ times higher than anticipated, based on their REGIONscores and the relationship between VARscore and mutation rate determined by Legendre et al. [25]. This was not unexpected because Legendre et al. had assessed mutation rates in haploid S. cerevisiae, whereas C. albicans is diploid [19]. Diploidy would be expected to increase TR mutation rates by providing opportunities for recombination between alleles [5].

Thus, our best estimate is a rate of $2 \times 10^{-2}$ mutations per generation in one of the 12,221 pcTR regions in the C. albicans genome, that is, one mutation every 44 divisions. Aside from TR indels, one mutation every 184 divisions is expected to occur across the entire $18 \mathrm{Mb}$ of protein-coding DNA (assuming a mutation rate of non-repetitive DNA of $3 \times 10^{-10}$ per bp $\left.[34,35]\right)$. This suggests that $\sim 80 \%$ of all mutations that occur in protein-coding DNA in C. albicans are pcTR mutations.

The strongest potential impact of mutations in pcTR regions would be frameshifts. However, approximately $85 \%$ of pcTR regions contained repeat stretches for which Tandem Repeats Finder reported units with lengths divisible by 3 (Table S3), indicating that changes in the number of repeat units will not lead to frameshifts. Tandem Repeats Finder does not report all possible unit sizes [26] and thus, for instance, three copies of a $4 \mathrm{bp}$ unit may form a $12 \mathrm{bp}$ unit, which may not be reported (an example, C2_02330W_B 1, is shown in Table 1). Thus, insertions and deletions of units in the remaining $15 \%$ of pcTR-regions will also often not affect the reading frame.

Most indels in pcTR regions are thus likely to alter the number of amino acid repeats. Expansions and contraction of amino acid repeat sequences can have considerable phenotypic consequences but in many cases the effect is probably minor [1,5,9]—as is also the case with other mutations [36]. However, in microbial species, even changes that affect fitness by less than $0.0001 \%$ are under selection [11-13].

\subsection{TR-ORFs Are Not Restricted to Particular Functions, Processes and Components}

Given the estimated impact of pcTR regions on mutation rate, it was of interest to determine what types of genes contain pcTR regions. To do so we used the GO annotation of the genome [24]. Figure 2 shows what percentage of all C. albicans genes associated with each GO term contains pcTR-regions. We expected that proteins involved in interaction with the host, in particular with cell surface-related roles, would often be encoded by TR-ORFS as well as regulatory proteins, as has been observed in other eukaryotes $[5,6,37]$. This was the case but the most striking outcome of this analysis was that for all but two GO terms more than $20 \%$ of all proteins assigned to a term were encoded by TR-ORFs. We identified the top $10 \%$ pcTR regions in terms of REGIONscore and repeated the analysis with the 300 genes that contained them but even these were fairly evenly distributed between GO categories (Figure 2)—although the difference in the frequency of high-scoring pcTR region-containing genes between some Process categories (adhesion, some forms of growth and biofilm formation) and the frequencies of such genes in the remaining categories was greater than when all pcTR regions were considered. 


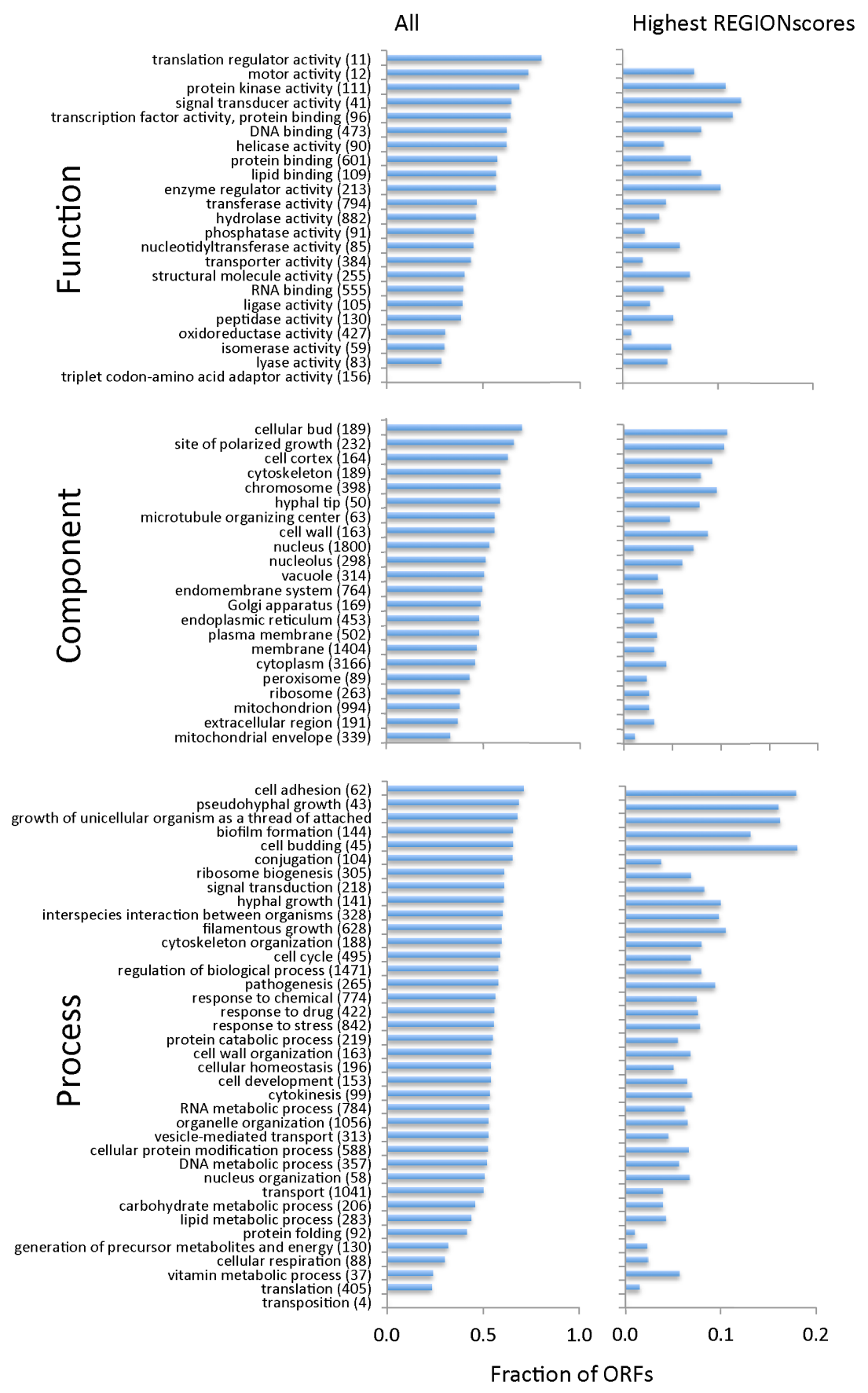

Figure 2. Frequency of TR-ORFs in different gene categories. Shown are the fractions of all genes in the C. albicans genome mapped to GO slim terms that are TR-ORFs. Numbers in brackets next to a term are the total number of genes in the genome mapped to that term. Shown on the left (All) are fractions of genes containing TR-ORFs. Fractions on the right (Highest REGIONscores) show the fraction, in each gene category, of TR-ORFS containing pcTRs whose REGIONscores are in the top $10 \%$ of all REGIONscores.

3.4. Amino Acid Repeat Units and Amino Acid Repeat Region Lengths Are under Selection and TR Mutation Generates Clade-Specific Protein Variants

A number of lines of evidence support the notion that pcTR regions are under selection in C. albicans, albeit to date only based on a small percentage of all pcTRs. These include (i) direct 
demonstration of a functional role of the pcTR region [38-42] (ii) functional impact of altered pcTR region length [43-46] (iii) elevated ratios of synonymous to nonsynonymous mutations in repeat units [47] and (iv) non-random distribution within pcTR regions of repeat units with nonsynonymous mutations $[16,17]$.

The species C. albicans is subdivided into clades that diverged 3-16 million years ago [48] and additional evidence that pcTR regions are under selection is the existence of clade-specific TR-ORF alleles or allele combinations-i.e., for a given pcTR-containing locus strains belonging to the same clade will often have the same combination of two pcTR region lengths [16,17,43,47,49-51]. Given that a large number of alleles and allele combinations exist for these TR-ORFs $[16,17,43,47,49-51]$, the rate of pcTR mutation and the time that has elapsed since clades diverged, the prevalence of one or two allele combinations in a clade is difficult to explain except by selection $[16,17,52]$; we have calculated that a few thousand years would have sufficed to eliminate clade-specificity of TR region length allele combinations if these were selectively neutral [17].

Clade-specific TR-ORFs and TR-ORF genotypes differing in pcTR lengths also offer an evolutionary explanation for the high frequency of pcTRs in C. albicans genes. Namely, pcTRs would have allowed C. albicans to evolve protein variants and variant combinations optimized for different genetic backgrounds (including epistatic interactions of clade-optimized alleles of a given TR-ORF with others). Such fine-tuning of over 10,000 proteins may have been difficult to achieve except through highly mutable pcTRs.

Optimizing proteins for a given genetic background by incorporating highly mutable regions into the ORFs does have a significant drawback though: continuing frequent mutation of the TR region will continue to produce new protein variants, suboptimal in this genetic background. The extent of the resulting disadvantage and the fitness advantage associated with having the "correct" clade-specific version can be estimated from the frequency of "incorrect" versions. This is possible because the frequency of allele combinations that differ from the optimal pcTR region lengths should be determined by the balance between the rate at which such allele combinations arise (i.e., the mutation rate of a TR region) and the rate at which they are removed by selection because their carriers are less fit (i.e., the selection coefficient [53]; genetic drift would not be expected to play a major role in determining their frequency given the large effective population size of microbial species [11-13]).

We have determined $[16,17]$ for two TR-ORFs, PNG2 and SSR1, that insertions and deletions of repeat units alter pcTR region lengths at a rate of 1.4 and $6 \times 10^{-5}$ per generation. We have also determined that $\sim 20 \%$ of isolates in the largest and genetically most homogeneous C. albicans clade $1[52,54]$ have allele combinations that differ from the predominant allele combinations. Thus, assuming that the unusual allele combinations are suboptimal (at least most of the time; see below), the selection coefficients of TR region length-altering changes for these two genes can be calculated as $3 \times 10^{-4}$ and $7 \times 10^{-5}$, respectively.

Assuming a frequency of $\sim 20 \%$ of suboptimal allele combinations across all 2936 TR-ORF loci in the genome and similar selection coefficients, pcTR mutation could reduce fitness by $20-80 \%-a$ considerable reduction. However, to put these numbers in perspective, they are equivalent to the typical fitness cost of a small number of deleterious point mutations $[55,56]$.

\subsection{Synonymous Mutations Reduce the Repetitiveness of Many TR Regions}

Synonymous mutations can reduce the repetitiveness and thus the mutation rate of DNA encoding an amino acid repeat. For example, the amino acid repeat SSSSSSSSSSSS, encoded, using only the codon TCC, as TCC TCC TCC TCC TCC TCC TCC TCC TCC TCC TCC TCC has a VARscore of 1.04. In contrast, the sequence TCC TCA TCT TCA TCT TCC TCC TCA TCT TCA TCT TCC encoding the same amino acid repeat but using three different codons, has a VARscore of -0.26 , equivalent to a predicted mutation rate 10 times lower [25] than the first sequence.

Since it is the amino acid repeat, rather than the DNA repeat that is under selection, one way to reduce the frequency at which suboptimal TR region alleles arise and thus the fitness cost associated 
with TR mutation, would be synonymous mutations. Even if pcTR regions initially had had no synonymous mutations, there would have been opportunity to acquire these during the evolutionary history of extant strains, judging by the frequency of point mutations present in their genomes: Bougnoux et al. found one polymorphic site every 34 bases in a set of 92 isolates [57] and the SC5314 genome contains one single nucleotide polymorphism (SNP) every 83 base pairs [58].

If reduction of the repetitiveness of a TR region encoding an amino acid repeat is selectively advantageous, synonymous mutations would be retained after they arose. In addition, the repeat unit that contained them would "spread" replacing other units by recombination and replication slippage events to further reduce repetitiveness of the region. Synonymous mutations can also carry a fitness cost, in the form of reduced efficiency of translation if the codon is rarely used [59]. However, given the abundance of TR regions and given that the age of many TR regions presumably exceeds the divergence of clades (as indicated by the existence of clade-specific alleles [16,17,43,47,49-51]), codon usage patterns should have co adapted if $C$. albicans had engaged in a strategy of using synonymous mutations to reduce the repetitiveness of pcTR regions.

We incorporated into TRANALYZER a feature that allowed us to assess if $C$. albicans uses synonymous mutations to reduce the repetitiveness of TR regions without altering the amino acid repeat. To do so, TRANALYZER eliminates and shuffles synonymous mutations in TR regions (see Methods and Figure 1b). It then recalculates the REGIONscores of these permutations in an attempt to find the highest REGIONscore.

Using this methodology, we found REGIONscores of theoretical permuted sequences that were higher than the observed scores for the same regions for 5220 of the 12,122 TR regions (Figure 3). The average reduction of REGIONscores by synonymous mutations in these 5220 TR regions was 0.16 . The average REGIONscore across all 12,122 TR regions was reduced by 0.21 through synonymous mutations, from a maximum possible average REGIONscore of -0.25 to its actual value of -0.45 . This correspond to a $25 \%$ reduction in the estimated genome-wide TR mutation rate [25].

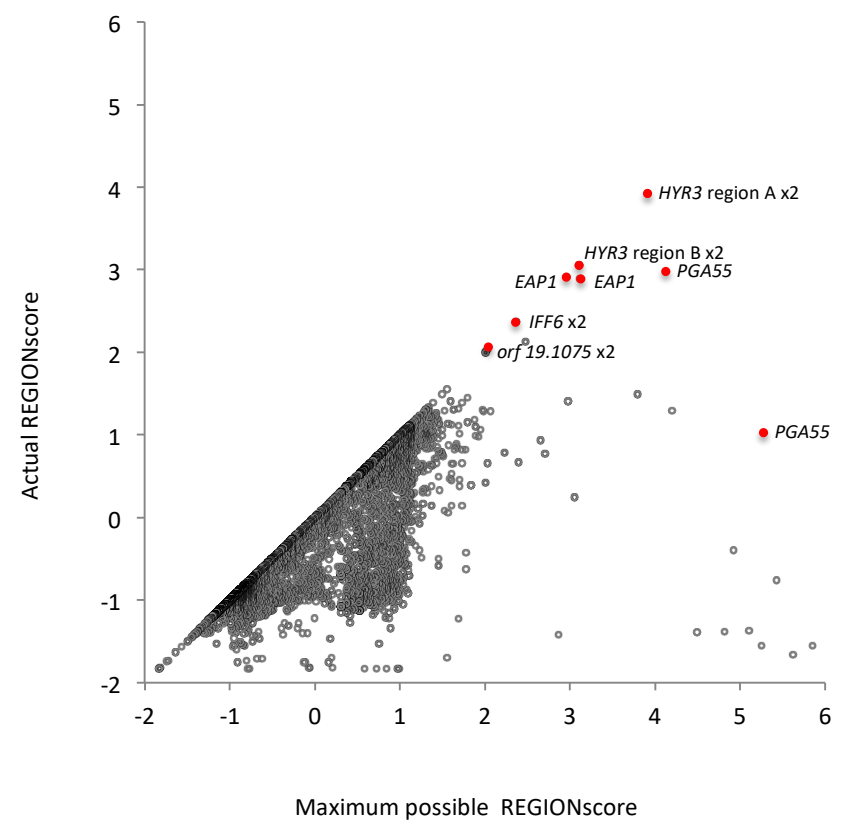

Figure 3. Plot of actual REGIONscores against highest possible REGIONscores for all C. albicans pcTR regions. pcTR regions with REGIONscores $>2$ for which allele distributions were investigated in a strain survey are marked in red and labelled. " $\times 2$ " indicates that the data point represents the values for both alleles in the SC5314 genome. 
Thus C. albicans widely uses synonymous mutations to reduce the repetitiveness and thus the mutation rate of pcTR regions.

It seemed of interest to explore if reduction of TR mutation rate by synonymous mutations might be a mechanism employed by eukaryotes in general. We therefore carried out the same type of analysis in two unrelated eukaryote species, in the plant Arabidopsis thaliana and in humans. We found that both of these species reduce repetitiveness of pcTR regions by synonymous mutations to a similar degree as C. albicans (Figure 4).

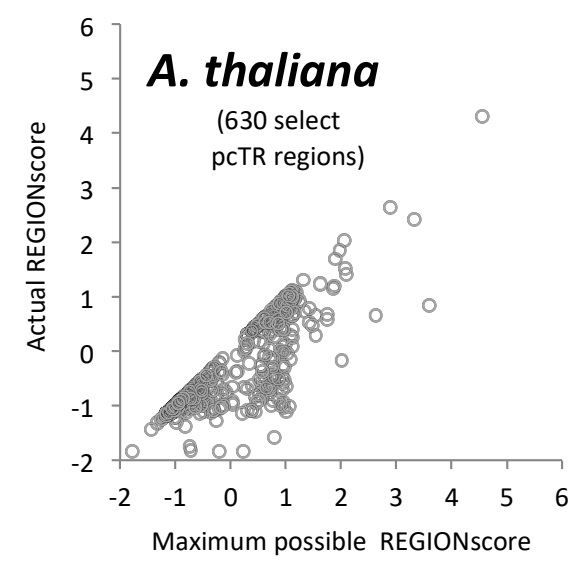

(a)

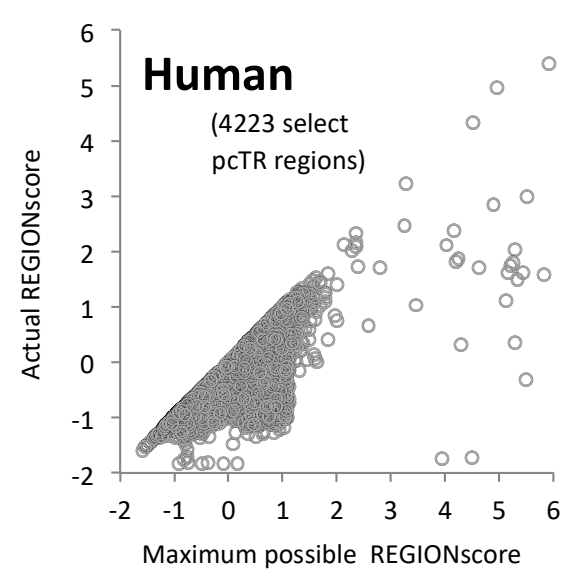

(b)

Figure 4. Plot of actual REGIONscores against highest possible REGIONscores for TR regions in (a) A. thaliana and (b) Homo sapiens. Values for a small selection of pcTR regions are shown.

We noticed that in these intron-rich genomes the location of pcTR regions relative to introns may be an additional mechanism for altering their mutation rates. In A. thaliana $50 \%$ of genes contained TR regions in their transcribed regions but only $40 \%$ of these TR regions were identical to, or contained, TR regions present in the CDS. In the human genome $90 \%$ of ORFs we analysed contained TR regions but only $1.6 \%$ of these TR regions were identical to, or contained, TR-regions present in the CDS (although this fraction was strongly size-dependent; in CDS' of 3-6 kb, for instance, $20.1 \%$ were not interrupted by introns and in CDS $>400 \mathrm{~kb} 0.2 \%$ ).

\subsection{TR-ORFS with Close-to-Maximal REGION Scores. Are They Contingency Genes?}

Clades seem well adapted to the range of habitats C. albicans is likely to encounter in the human host, apparently its primary habitats $[19,20]$. Within a clade, clade-specific alleles appear to be prevalent for the vast majority of loci, regardless of body location and type of individual the strains are isolated from $[16,17,43,47,49-52,60]$. The available evidence suggests only limited specialization of clades towards particular types of individuals, body locations or interaction with the host (pathogenic versus commensal) $[33,60,61]$. Genetically indistinguishable strains can often be found in different body sites of an individual $[29,62]$ and opportunities for generating specialist lineages by parasexual recombination are exceedingly rare [22,63]. All of this indicates that selection, including selection of pcTR region alleles, generally favours broadly adapted general-purpose genotypes [33] and that the clades represent different evolutionary paths towards such genotypes.

However, even if long-term success depends on maintaining a general-purpose genotype, transient reversible alterations can assist in adaptation to particular habitats $[64,65]$. Given their high mutation rates and the large size of populations of $C$. albicans in the human host [19] TR-ORFs would offer C. albicans an opportunity to slightly and transiently, alter its genotype. From the constantly replenished pool of TR-ORF variants, some might be favoured by selection in a particular habitat. 
These may, for a time, become the predominant variant in that habitat, later to be replaced, from the pool, by the original general-purpose allele.

In addition, there could be TR-ORFs for which no generally optimal alleles exist, including perhaps some for which fitness benefits arise from constant change: Contingency genes which put the population in a state to exploit transient habitats and to evade host responses $[8,18,66-68]$. These could for instance generate, through TR mutations, habitat-specific adhesins $[39,43,44,69]$ or alterations of the wall $[16,46,47,50,70,71]$. The latter could affect host recognition [72] similar to the mechanism used by Plasmodium falciparum to elude the host response [73]. Provided that the ability to cause disease on occasions, or survival of the host response triggered by causing disease, is selectively sufficiently advantageous [52,74], some TR-contingency genes could have evolved to have roles in candidiasis, that is, when C. albicans' interaction with the host changes from commensal to pathogenic.

If alternative alleles of a TR-ORF assist in short-term adaptation then it is important to maintain a pool of such alleles by mutation. Thus, in such TR-ORFs mutation should have greater net benefits than in other TR-ORFs. A high REGIONscore and codon usage that maximizes REGIONscore could thus be an indication that mutation of a pcTR region is used for transient adaptation or evasion of host responses and that the TR-ORF that contains the region is a contingency gene. We identified 19 pcTR regions that on this basis could be considered most likely to have a role in short-term adaptation. They are the pcTRs with the highest REGIONscores, all $\geq 2$, largely undiminished by synonymous mutations (Figure 3; Table S3).

The19 pcTR regions were located in nine different genes (Table S3). Interestingly, for one of them, C2_08980C (PGA 55), the repeat region of only one copy of the gene is part of the set. In the same region of the second copy the actual REGIONscore is reduced to 1.0 (from a maximum score of 5.3 achievable if only one type of codon per amino acid were used; Figure 3). Also, several of the nine TR-ORFs contained additional pcTR regions with REGIONscores significantly reduced by synonymous mutations (Table S3).

Four of the nine genes are GO-annotated as encoding proteins located on the cell surface; a 14-fold overrepresentation (GO Finder $p=0.001$ ) Five are annotated as encoding adhesins or putative adhesins, far in excess of the genome-wide frequency of genes GO-annotated as involved in adhesion ("Cell adhesion"; $1 \%$; however, while adhesion is described as their likely role, only one of them is actually formally linked to this GO term). The TR regions of two of the genes (IFF6 and HYR3) encode very similar amino acid repeats and the proteins have $38 \%$ sequence identity ( $E$ value of $3 \times 10^{-102}$ in a Blast P search against all predicted proteins in the SC5314 genome assembly).

We screened five of the genes for TR region length polymorphisms in a collection of 58 strains, both commensal and disease-causing, representative of the different clades and isolated from different body sites (Table S1; Figure 5). Because it is difficult to assemble long DNA repeat regions by sequencing, we did so by gel electrophoresis. A drawback of this methodology is that alleles of the same length cannot further be differentiated based on sequence differences and that small difference in size between alleles could be missed. Nevertheless, it can provide some insights into selective restrains of repeat region lengths.

Genetic background did apparently play a role in selection of region lengths even in these highly mutable pcTR-regions. We assessed this by comparing lengths in 35 strains in the largest and genetically homogenous clade, clade $1[33,61]$ with those in 23 strains from the remainder of the species. For all pcTR regions, one region length combination genotype was significantly more frequent in clade 1 strains compared to other strains (Fisher's exact test, $p$ values of $0.006-1.5 \times 10^{-6}$; Figure 5; Table S1).

The number of different alleles and genotypes observable differed considerable between the pcTR-regions. Most restricted in this regard was the pcTR region in C2_09130C (IFF6): 70\% of all alleles we assessed were of the same apparent size and only six IFF6 genotypes were distinguishable. Also, notably, we never saw two different allele sizes in the same strain. The HYR3 region B, encoding a series of very similar amino acid repeats, seemed under similar restraints as was the HYR3 region 
A. All three regions not only had a limited number of alleles but the different alleles were also fairly similar in size.

A possible explanation of these restraints in spite of high REGIONscores undiminished by codon usage could be that the observed alleles are strongly selected under most circumstances but that there are occasions where the existence of alternate alleles in the population is crucial to survival, selecting for high mutation rates. We found some evidence supporting this hypothesis when comparing clade 1 isolates from different sources, including the bloodstream, an environment rarely encountered by C. albicans [75]. Of eight clade 1 bloodstream isolates, three were homozygous for an $825 \mathrm{bp}$ pcTR region 2 allele, compared to one out of 27 isolates from other sites ( $p=0.03$; Fisher's exact test).

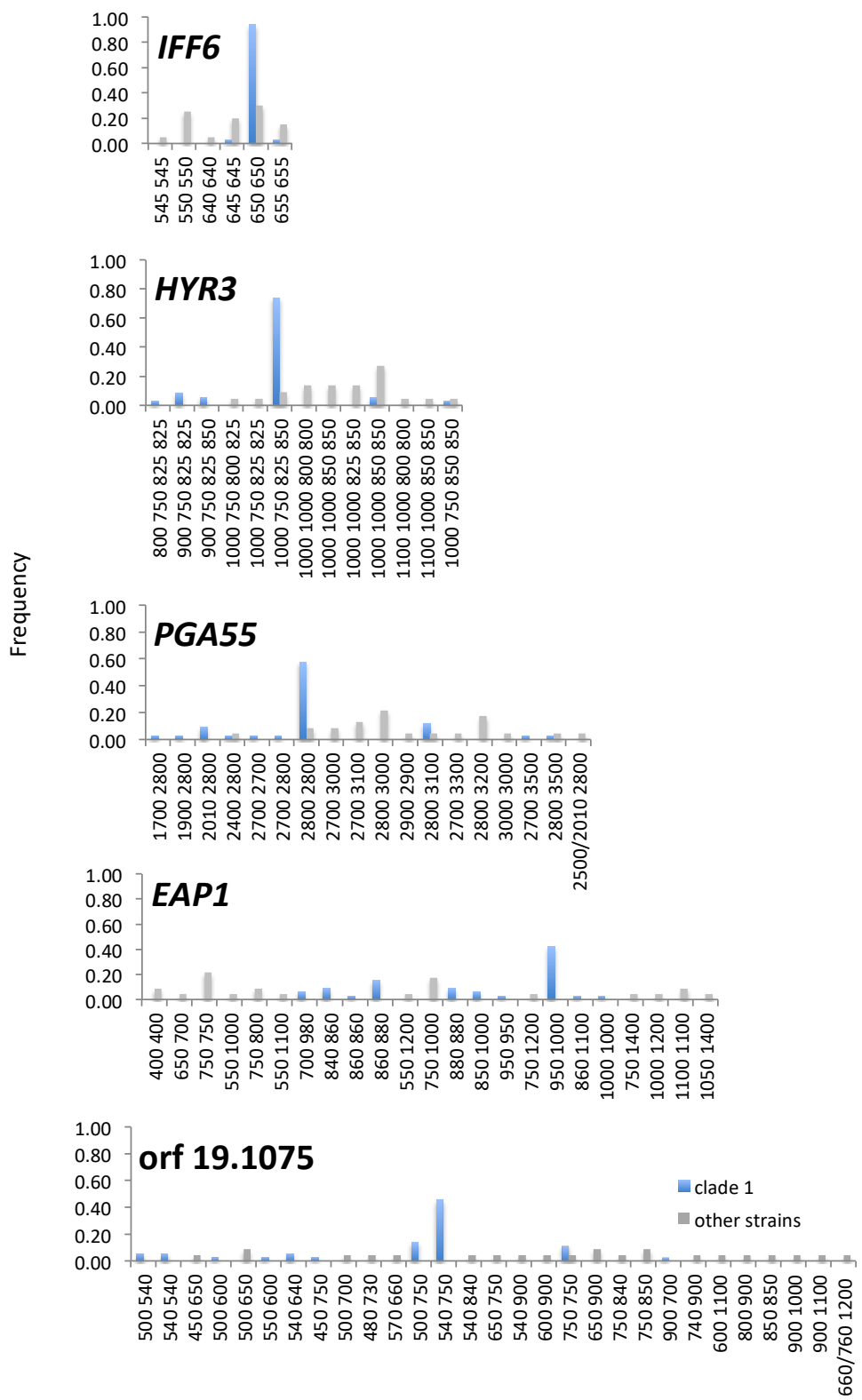

Figure 5. Allele combination frequencies of TR regions with high VARscores in five C. albicans genes in clade 1 strains (blue bars) and other strains (grey bars). Numbers indicate the size of PCR products amplified from the gene. In cases in which only one product size was obtained it was assumed that the strain had two alleles with identical TR region lengths. For $H Y R 3$ allele types include both TRregion A (first pair of numbers) and TR region B (second pair of numbers). 
The pcTR region of C2_08980C (PGA55), another likely adhesin, had a somewhat wider range of allele sizes. However, the diversity of length-based genotypes, 18 types, was comparable to that of other TR-ORFs with much lower REGIONscores (SSR1; 41 in 131 strains and PNG2; 23 alleles in 80 strains $[16,17])$. Thus, the PGA55 pcTR region lengths, since predicted to be more mutable, may be under more selective constrains than those of the aforementioned genes with lower REGIONscores.

When considering the contrast between the observed diversity of alleles and the expected diversity of these pcTR regions, based on their REGIONscores, it must be borne in mind that REGIONscores only provide estimates and cannot exactly predict how frequently indels generate new alleles. However, all of the above pcTR regions are exceptionally long (631-2283 bp; Table S3) and thus expected to be exceptionally mutable.

Length polymorphisms in the pcTR region in the adhesin EAP1 (C2_09530W) and in the other remaining pcTR region, in C2_09130C (an uncharacterized ORF), are most in line with expectations for those of contingency genes frequently involved in short term adaptation to transient habitats, namely a wide range of allele sizes and a comparatively low frequency of the prevalent genotype. For EAP1 we also found some indication of possible roles in habitat-specific adaptation within clade 1 . The frequency of alleles sized $840-880$ bp was correlated with the habitats from which strains had been obtained. They were present in all of seven bloodstream isolates, in seven of 13 commensal isolates but in only one of 13 isolates from superficial infections (Chi square test, $p<0.01$ ).

\section{Conclusions}

Our data support the notion that in C. albicans highly mutable pcTRs allow the evolution of protein variants with properties sufficiently distinct to be under selection. This has generated protein variants that are optimized for specific genetic backgrounds. However, the high mutation rates of pcTRs are likely to represent a considerable ongoing fitness cost. C. albicans apparently reduces this cost by "down-regulating" the mutation rates through incorporation of synonymous mutations and does so to different degrees in different TR-ORFs.

Even among TR-ORFs with high REGIONscores, largely undiminished by synonymous mutations we did not find any for which habitat is a stronger driver of selection than the genetic background. Thus, apparently even the most mutable pcTRs encode clade-specific protein variants optimized for performance across many habitats. This is perhaps not unexpected, given that $C$. albicans' almost exclusively clonal mode of propagation [22] and a dynamic human C. albicans microbiome [62] should favour generally adapted genotypes over specialists.

Our small survey reported here and earlier work [16] suggests that, superimposed on this genetic background-driven selection, some additional selection aimed at adaptation to specific habitats can occur. One of the strongest indications is the contrast between high REGIONscores and a very limited number of alleles in our surveys of IFF6 and HYR3. It suggests that under yet-to-be identified circumstances the existence of alternate alleles is crucial. For HYR3 and some other TR-ORFs our (very limited) information on the origin of isolates suggests that the transition to pathogenesis might be one situation that selects for alleles different from those optimal in commensalism. However, given that C. albicans causes disease so rarely, maintaining a pool of alternate alleles is probably more important in other situations. The colonization of the newborn from its mother [76] would be a likely one. Age-dependent changes in clade prevalence [60] suggest that the genotypes optimal for colonization of a newborn are different from those optimal for colonization of its mother. The ability to increase the chances of colonization of a new host should constitute a powerful selective pressure for maintaining a high mutation rate in a pcTR region. Further studies on additional TR regions and additional strains with detailed information regarding the hosts and the sites from which they were isolated are needed to elucidate to what degree TR mutation assists in habitat-specific adaptation.

The Red Queen hypothesis states that organisms must constantly evolve to keep up with their competitors, one of the reasons why achieving the lowest possible mutation rate is not necessarily advantageous $[77,78]$. Aside from offering an opportunity for optimizing alleles, introduction of pcTRs 
may be selectively advantageous, in spite of the constant cost of generation of suboptimal alleles, by maintaining a mutation rate sufficiently high for competition in the Red Queen's race. This may be another major reason for the high frequency of pcTRs in the $C$. albicans genome.

One considerable advantage of pcTRs over adjusting mutation rates by altering of DNA repair and proofreading mechanisms [78] is that through the incorporation or removal of synonymous mutations the mutation rate can be adjusted for individual pcTR regions. C. albicans is apparently not unique among eukaryotes in employing this strategy. When we analysed the genome of Arabidopsis thaliana and the human genome we found that both of these species reduce repetitiveness of pcTR regions by synonymous mutations to a similar degree as C. albicans (Figure 4). In such intron-rich genomes the location of TR regions relative to introns may contribute additional facets to the evolutionary biology of TR regions. Interruption of amino acid repeat-encoding DNA by introns could reduce the rate at which new protein variants arise, while TR region expansion within introns can generate new protein variants, in addition to a raft of other effects [79,80]. Given the high percentage of TR regions in untranslated parts of transcribed DNA, in many genomes TR mutation may exert much of its phenotypic effect by altering introns and $5^{\prime}$ and $3^{\prime}$ untranslated regions [79-82].

Current attempts to understand the evolutionary forces that determine mutation rate are largely focused on point mutations [78]. Our results for C. albicans add to the growing evidence that pcTR mutation may deserve more consideration as a driver of mutational advance.

Supplementary Materials: The following are available online at http:/ / www.mdpi.com/2309-608X/4/3/78/s1, Table S1: Strains and Alleles, Table S2: Primers, Table S3: REGIONscores.

Author Contributions: M.W. wrote and operated the TRANALYZER software, N.Z. designed and carried out molecular biology experiments. J.S. developed the concept underlying the analyses, interpreted the data, wrote the paper and generated the figures.

Funding: This research was funded by the Massey University Research Fun and the Maurice and Phyllis Paykel Trust.

Acknowledgments: We thank Dave Wheeler for retrieving for us human gene sequences and Murray Cox, Paul Dijkwel and Peter Lipke for valuable comments on the manuscript

Conflicts of Interest: The authors declare no conflict of interest.

\section{References}

1. Gemayel, R.; Vinces, M.D.; Legendre, M.; Verstrepen, K.J. Variable tandem repeats accelerate evolution of coding and regulatory sequences. Annu. Rev. Genet. 2010, 44, 445-477. [CrossRef] [PubMed]

2. Ahmed, M.; Liang, P. Transposable elements are a significant contributor to tandem repeats in the human genome. Comp. Funct. Genom. 2012, 2012, 947089. [CrossRef] [PubMed]

3. Taylor, J.S.; Breden, F. Slipped-strand mispairing at noncontiguous repeats in poecilia reticulata: A model for minisatellite birth. Genetics 2000, 155, 1313-1320. [PubMed]

4. Haber, J.E.; Louis, E.J. Minisatellite origins in yeast and humans. Genomics 1998, 48, 132-135. [CrossRef] [PubMed]

5. Gemayel, R.; Cho, J.; Boeynaems, S.; Verstrepen, K.J. Beyond junk-variable tandem repeats as facilitators of rapid evolution of regulatory and coding sequences. Genes 2012, 3, 461-480. [CrossRef] [PubMed]

6. Gemayel, R.; Chavali, S.; Pougach, K.; Legendre, M.; Zhu, B.; Boeynaems, S.; van der Zande, E.; Gevaert, K.; Rousseau, F.; Schymkowitz, J.; et al. Variable glutamine-rich repeats modulate transcription factor activity. Mol. Cell 2015, 59, 615-627. [CrossRef] [PubMed]

7. Jansen, A.; Gemayel, R.; Verstrepen, K.J. Unstable microsatellite repeats facilitate rapid evolution of coding and regulatory sequences. Genome Dyn. 2012, 7, 108-125. [PubMed]

8. Deitsch, K.W.; Lukehart, S.A.; Stringer, J.R. Common strategies for antigenic variation by bacterial, fungal and protozoan pathogens. Nat. Rev. Microbiol. 2009, 7, 493-503. [CrossRef] [PubMed]

9. Simon, M.; Hancock, J.M. Tandem and cryptic amino acid repeats accumulate in disordered regions of proteins. Genome Biol. 2009, 10, R59. [CrossRef] [PubMed]

10. Albà, M.M.; Guigó, R. Comparative analysis of amino acid repeats in rodents and humans. Genome Res. 2004, 14, 549-554. [CrossRef] [PubMed] 
11. Lynch, M.; Conery, J.S. The origins of genome complexity. Science 2003, 302, 1401-1404. [CrossRef] [PubMed]

12. Tsai, I.J.; Bensasson, D.; Burt, A.; Koufopanou, V. Population genomics of the wild yeast Saccharomyces paradoxus: Quantifying the life cycle. Proc. Natl. Acad. Sci. USA 2008, 105, 4957-4962. [CrossRef] [PubMed]

13. Charlesworth, B. Fundamental concepts in genetics: Effective population size and patterns of molecular evolution and variation. Nat. Rev. Genet. 2009, 10, 195-205. [CrossRef] [PubMed]

14. Sim, K.L.; Creamer, T.P. Protein simple sequence conservation. Proteins 2004, 54, 629-638. [CrossRef] [PubMed]

15. Hoyer, L.; Fundyga, R.; Hecht, J.E.; Kapteyn, J.C.; Klis, F.M.; Arnold, J. Characterization of agglutinin-like sequence genes from non-albicans Candida and phylogenetic analysis of the als family. Genetics 2001, 157, 1555-1567. [PubMed]

16. Zhou, Z.; Jordens, Z.; Zhang, S.; Zhang, N.; Schmid, J. Highly mutable tandem DNA repeats generate a cell wall protein variant more frequent in disease-causing Candida albicans isolates than in commensal isolates. PLoS ONE 2017, 12, e0180246. [CrossRef] [PubMed]

17. Zhang, N.; Cannon, R.D.; Holland, B.; Patchett, M.; Schmid, J. Impact of genetic background on allele selection in a highly mutable Candida albicans gene, PNG2. PLoS ONE 2010, 5, e9614. [CrossRef]

18. Zhou, K.; Aertsen, A.; Michiels, C.W. The role of variable DNA tandem repeats in bacterial adaptation. FEMS Microbiol. Rev. 2014, 38, 119-141. [CrossRef] [PubMed]

19. Odds, F.C. Candida and Candidosis, 2nd ed.; Bailliere Tindall: London, UK, 1988.

20. Skinner, C.E.; Fletcher, D.W. A review of the genus Candida. Bacteriol. Rev. 1960, 24, 397-416. [PubMed]

21. Braun, B.R.; van het Hoog, M.; d’Enfert, C.; Martchenko, M.; Dungan, J.; Kuo, A.; Inglis, D.O.; Uhl, M.A.; Hogues, H.; Berriman, M.; et al. A human-curated annotation of the Candida albicans genome. PLoS Genet. 2005, 1, 36-57. [CrossRef] [PubMed]

22. Schmid, J.; Magee, P.T.; Holland, B.R.; Zhang, N.; Cannon, R.D.; Magee, B.B. Last hope for the doomed? Thoughts on the importance of a parasexual cycle for the yeast Candida albicans. Curr. Genet. 2015, 62, 81-85. [CrossRef] [PubMed]

23. Bruno, V.M.; Wang, Z.; Marjani, S.L.; Euskirchen, G.M.; Martin, J.; Sherlock, G.; Snyder, M. Comprehensive annotation of the transcriptome of the human fungal pathogen Candida albicans using RNA-seq. Genome Res. 2010, 20, 1451-1458. [CrossRef] [PubMed]

24. Arnaud, M.; Inglis, D.; Skrzypek, M.; Binkley, J.; Shah, P.; Wymore, F.; Binkley, G.; Miyasato, S.; Simison, M.; Sherlock, G. Candida Genome Database. Available online: http:/ / www.candidagenome.org/ (accessed on 15 April 2018).

25. Legendre, M.; Pochet, N.; Pak, T.; Verstrepen, K.J. Sequence-based estimation of minisatellite and microsatellite repeat variability. Genome Res. 2007, 17, 1787-1796. [CrossRef] [PubMed]

26. Benson, G. Tandem repeats finder: A program to analyse DNA sequences. Nucleic Acids Res. 1999, 27, 573-580. [CrossRef] [PubMed]

27. Holland, B.R.; Schmid, J. Selecting representative model micro-organisms. BMC Microbiol. 2005, 5, 26. [CrossRef] [PubMed]

28. Schmid, J.; Voss, E.; Soll, D.R. Computer-assisted methods for assessing strain relatedness in Candida albicans by fingerprinting with the moderately repetitive sequence Ca3. J. Clin. Microbiol. 1990, 28, 1236-1243. [PubMed]

29. Soll, D.R.; Galask, R.; Schmid, J.; Hanna, C.; Mac, K.; Morrow, B. Genetic dissimilarity of commensal strains of Candida spp. Carried in different anatomical locations of the same healthy women. J. Clin. Microbiol. 1991, 29, 1702-1710. [PubMed]

30. Schmid, J.; Odds, F.C.; Wiselka, M.J.; Nicholson, K.G.; Soll, D.R. Genetic similarity and maintenance of Candida albicans strains from a group of aids patients, demonstrated by DNA fingerprinting. J. Clin. Microbiol. 1992, 30, 935-941. [PubMed]

31. Schmid, J.; Rotman, M.; Reed, B.; Pierson, C.L.; Soll, D.R. Genetic similarity of Candida albicans strains from vaginitis patients and their partners. J. Clin. Microbiol. 1993, 31, 39-46. [PubMed]

32. Schmid, J.; Tay, Y.P.; Wan, L.; Carr, M.; Parr, D.; McKinney, W. Evidence for nosocomial transmission of Candida albicans obtained by ca3 fingerprinting. J. Clin. Microbiol. 1995, 33, 1223-1230. [PubMed] 
33. Schmid, J.; Herd, S.; Hunter, P.R.; Cannon, R.D.; Yasin, M.S.M.; Samad, S.; Carr, M.; Parr, D.; McKinney, W.; Schousboe, M.; et al. Evidence for a general-purpose genotype in Candida albicans, highly prevalent in multiple geographic regions, patient types and types of infection. Microbiology 1999, 145, 2405-2414. [CrossRef] [PubMed]

34. Lynch, M.; Sung, W.; Morris, K.; Coffey, N.; Landry, C.R.; Dopman, E.B.; Dickinson, W.J.; Okamoto, K.; Kulkarni, S.; Hartl, D.L.; et al. A genome-wide view of the spectrum of spontaneous mutations in yeast. Proc. Natl. Acad. Sci. USA 2008, 105, 9272-9277. [CrossRef] [PubMed]

35. Gomez-Raja, J.; Larriba, G. Comparison of two approaches for identification of haplotypes and point mutations in Candida albicans and saccharomyces cerevisiae. J. Microbiol. Methods 2013, 94, 47-53. [CrossRef] [PubMed]

36. Abdullah, T.; Faiza, M.; Pant, P.; Rayyan Akhtar, M.; Pant, P. An analysis of single nucleotide substitution in genetic codons-probabilities and outcomes. Bioinformation 2016, 12, 98-104. [CrossRef] [PubMed]

37. Young, E.T.; Sloan, J.S.; Van Riper, K. Trinucleotide repeats are clustered in regulatory genes in saccharomyces cerevisiae. Genetics 2000, 154, 1053-1068. [PubMed]

38. Staab, J.F.; Bahn, Y.S.; Tai, C.H.; Cook, P.F.; Sundstrom, P. Expression of transglutaminase substrate activity on Candida albicans germ tubes through a coiled, disulfide-bonded n-terminal domain of Hwp1 requires c-terminal glycosylphosphatidylinositol modification. J. Biol. Chem. 2004, 279, 40737-40747. [CrossRef] [PubMed]

39. Li, F.; Palecek, S.P. Distinct domains of the Candida albicans adhesin Eap1p mediate cell-cell and cell-substrate interactions. Microbiology 2008, 154, 1193-1203. [CrossRef] [PubMed]

40. Chin, C.; Lai, W.-C.; Lee, T.-L.; Tseng, T.-L.; Shieh, J.-C. Dissection of the Candida albicans Cdc4 protein reveals the involvement of domains in morphogenesis and cell flocculation. J. Biomed. Sci. 2013, 20, 97. [CrossRef] [PubMed]

41. Liu, X.; Nie, X.; Ding, Y.; Chen, J. Asc1, a wd-repeat protein, is required for hyphal development and virulence in Candida albicans. Acta Biochim. Biophys. Sin. 2010, 42, 793-800. [CrossRef] [PubMed]

42. Boisrame, A.; Cornu, A.; Da Costa, G.; Richard, M.L. Unexpected role for a serine/threonine-rich domain in the Candida albicans Iff protein family. Eukaryot. Cell 2011, 10, 1317-1330. [CrossRef] [PubMed]

43. Oh, S.-H.; Cheng, G.; Nuessen, J.A.; Jajko, R.; Yeater, K.M.; Zhao, X.; Pujol, C.; Soll, D.R.; Hoyer, L.L. Functional specificity of Candida albicans Als3p proteins and clade specificity of ALS3 alleles discriminated by the number of copies of the tandem repeat sequence in the central domain. Microbiology 2005, 151, 673-681. [CrossRef] [PubMed]

44. Rauceo, J.M.; De Armond, R.; Otoo, H.; Kahn, P.C.; Klotz, S.A.; Gaur, N.K.; Lipke, P.N. Threonine-rich repeats increase fibronectin binding in the Candida albicans adhesin Als5p. Eukaryot. Cell 2006, 5, 1664-1673. [CrossRef] [PubMed]

45. Sumita, T.; Yoko-o, T.; Shimma, Y.-i.; Jigami, Y. Comparison of cell wall localization among pir family proteins and functional dissection of the region required for cell wall binding and bud scar recruitment of Pir1p. Eukaryot. Cell 2005, 4, 1872-1881. [CrossRef] [PubMed]

46. Sampaio, P.; Nogueira, E.; Loureiro, A.; Delgado-Silva, Y.; Correia, A.; Pais, C. Increased number of glutamine repeats in the c-terminal of Candida albicans Rlm1p enhances the resistance to stress agents. Antonie Van Leeuwenhoek 2009, 96, 395-404. [CrossRef] [PubMed]

47. Zhang, N.; Harrex, A.L.; Holland, B.R.; Fenton, L.E.; Cannon, R.D.; Schmid, J. Sixty alleles of the ALS7 open reading frame in Candida albicans: Als7 is a hypermutable contingency locus. Genome Res. 2003, 13, 2005-2017. [CrossRef] [PubMed]

48. Lott, T.J.; Fundyga, R.E.; Kuykendall, R.J.; Arnold, J. The human commensal yeast, Candida albicans, has an ancient origin. Fungal Genet. Biol. 2005, 42, 444-451. [CrossRef] [PubMed]

49. Lott, T.J.; Holloway, B.P.; Logan, D.A.; Fundyga, R.; Arnold, J. Towards understanding the evolution of the human commensal yeast Candida albicans. Microbiology 1999, 145, 1137-1143. [CrossRef] [PubMed]

50. Zhao, X.; Oh, S.H.; Jajko, R.; Diekema, D.J.; Pfaller, M.A.; Pujol, C.; Soll, D.R.; Hoyer, L.L. Analysis of ALS5 and ALS6 allelic variability in a geographically diverse collection of Candida albicans isolates. Fungal Genet. Biol. 2007, 44, 1298-1309. [CrossRef] [PubMed]

51. MacCallum, D.M.; Castillo, L.; Nather, K.; Munro, C.A.; Brown, A.J.; Gow, N.A.; Odds, F.C. Property differences among the four major Candida albicans strain clades. Eukaryot. Cell. 2009, 8, 373-387. [CrossRef] [PubMed] 
52. Zhang, N.; Upritchard, J.E.; Holland, B.R.; Fenton, L.E.; Ferguson, M.M.; Cannon, R.D.; Schmid, J. Distribution of mutations distinguishing the most prevalent disease-causing Candida albicans genotype from other genotypes. Infect. Genet. Evol. 2009, 9, 493-500. [CrossRef] [PubMed]

53. Ridley, M. Evolution; Blackwell Science: Cambridge, MA, USA, 1996.

54. Tavanti, A.; Davidson, A.D.; Fordyce, M.J.; Gow, N.A.R.; Maiden, M.C.J.; Odds, F.C. Population structure and properties of Candida albicans, as determined by multilocus sequence typing. J. Clin. Microbiol. 2005, 43, 5601-5613. [CrossRef] [PubMed]

55. Eyre-Walker, A.; Keightley, P.D. The distribution of fitness effects of new mutations. Nat. Rev. Genet. 2007, 8, 610. [CrossRef] [PubMed]

56. Wloch, D.M.; Szafraniec, K.; Borts, R.H.; Korona, R. Direct estimate of the mutation rate and the distribution of fitness effects in the yeast Saccharomyces cerevisiae. Genetics 2001, 159, 441-452. [PubMed]

57. Bougnoux, M.E.; Tavanti, A.; Bouchier, C.; Gow, N.A.; Magnier, A.; Davidson, A.D.; Maiden, M.C.; D'Enfert, C.; Odds, F.C. Collaborative consensus for optimized multilocus sequence typing of Candida albicans. J. Clin. Microbiol. 2003, 41, 5265-5266. [CrossRef] [PubMed]

58. Forche, A.; Magee, P.T.; Magee, B.B.; May, G. Genome-wide single-nucleotide polymorphism map for Candida albicans. Eukaryot. Cell 2004, 3, 705-714. [CrossRef] [PubMed]

59. Hanson, G.; Coller, J. Codon optimality, bias and usage in translation and mRNA decay. Nat. Rev. Mol Cell. Biol. 2018, 19, 20-30. [CrossRef] [PubMed]

60. Schmid, J.; Tortorano, A.M.; Jones, G.; Lazzarini, C.; Zhang, N.; Bendall, M.J.; Cogliati, M.; Wattimena, S.; Klingspor, L.; ECMM_survey_participants; et al. Increased mortality in young candidemia patients associated with presence of a Candida albicans general-purpose genotype. J. Clin. Microbiol. 2011, 49, 3250-3256. [CrossRef] [PubMed]

61. Odds, F.C.; Bougnoux, M.E.; Shaw, D.J.; Bain, J.M.; Davidson, A.D.; Diogo, D.; Jacobsen, M.D.; Lecomte, M.; Li, S.Y.; Tavanti, A.; et al. Molecular phylogenetics of Candida albicans. Eukaryot. Cell 2007, 6, 1041-1052. [CrossRef] [PubMed]

62. Zhang, N.; Wheeler, D.; Truglio, M.; Lazzarini, C.; Upritchard, J.; McKinney, W.; Rogers, K.; Prigitano, A.; Tortorano, A.M.; Cannon, R.D.; et al. Multi-locus next-generation sequence typing of DNA extracted from pooled colonies detects multiple unrelated Candida albicans strains in a significant proportion of patient samples. Front. Microbiol. 2018, 9. [CrossRef] [PubMed]

63. Zhang, N.; Magee, B.B.; Magee, P.T.; Holland, B.R.; Rodrigues, E.; Holmes, A.R.; Cannon, R.D.; Schmid, J. Selective advantages of a parasexual cycle for the yeast Candida albicans. Genetics 2015, 200, 1117-1132. [CrossRef] [PubMed]

64. Massicotte, R.; Angers, B. General-purpose genotype or how epigenetics extend the flexibility of a genotype. Genet. Res. Int. 2012, 2012, 317175. [CrossRef] [PubMed]

65. Stearns, S.C. The evolutionary significance of phenotypic plasticityphenotypic sources of variation among organisms can be described by developmental switches and reaction norms. Bioscience 1989, 39, 436-445. [CrossRef]

66. Moxon, R.; Bayliss, C.; Hood, D. Bacterial contingency loci: The role of simple sequence DNA repeats in bacterial adaptation. Annu. Rev. Genet. 2006, 40, 307-333. [CrossRef] [PubMed]

67. Verstrepen, K.J.; Reynolds, T.B.; Fink, G.R. Origins of variation in the fungal cell surface. Nat. Rev. Microbiol. 2004, 2, 533-540. [CrossRef] [PubMed]

68. Virji, M. Pathogenic neisseriae: Surface modulation, pathogenesis and infection control. Nat. Rev. Microbiol. 2009, 7, 274-286. [CrossRef] [PubMed]

69. Zhao, X.M.; Oh, S.H.; Hoyer, L.L. Unequal contribution of ALS9 alleles to adhesion between Candida albicans and human vascular endothelial cells. Microbiology 2007, 153, 2342-2350. [CrossRef] [PubMed]

70. Hoyer, L.L.; Scherer, S.; Shatzman, A.R.; Livi, G.P. Candida albicans ALS1: Domains related to a Saccharomyces cerevisiae sexual agglutinin separated by a repeating motif. Mol. Microbiol. 1995, 15, 39-54. [CrossRef] [PubMed]

71. Hoyer, L. The als gene family of Candida albicans. Trends Microbiol. 2001, 9, 176-180. [CrossRef]

72. Richardson, J.P.; Moyes, D.L. Adaptive immune responses to Candida albicans infection. Virulence 2015, 6, 327-337. [CrossRef] [PubMed]

73. Verstrepen, K.J.; Fink, G.R. Genetic and epigenetic mechanisms underlying cell-surface variability in protozoa and fungi. Annu. Rev. Genet. 2009, 43, 1-24. [CrossRef] [PubMed] 
74. Rivera-Perez, J.I.; Gonzalez, A.A.; Toranzos, G.A. From evolutionary advantage to disease agents: Forensic reevaluation of host-microbe interactions and pathogenicity. Microbiol. Spectrum 2017, 5. [CrossRef] [PubMed]

75. Diekema, D.J.; Messer, S.A.; Brueggemann, A.B.; Coffman, S.L.; Doern, G.V.; Herwaldt, L.A.; Pfaller, M.A. Epidemiology of candidemia: 3-year results from the emerging infections and the epidemiology of Iowa organisms study. J. Clin. Microbiol. 2002, 40, 1298-1302. [CrossRef] [PubMed]

76. Filippidi, A.; Galanakis, E.; Maraki, S.; Galani, I.; Drogari-Apiranthitou, M.; Kalmanti, M.; Mantadakis, E.; Samonis, G. The effect of maternal flora on Candida colonisation in the neonate. Mycoses 2014, 57, 43-48. [CrossRef] [PubMed]

77. Brockhurst, M.A.; Chapman, T.; King, K.C.; Mank, J.E.; Paterson, S.; Hurst, G.D. Running with the red queen: The role of biotic conflicts in evolution. Proc. Biol. Sci. 2014, 281, 20141382. [CrossRef] [PubMed]

78. Lynch, M.; Ackerman, M.S.; Gout, J.F.; Long, H.; Sung, W.; Thomas, W.K.; Foster, P.L. Genetic drift, selection and the evolution of the mutation rate. Nat. Rev. Genet. 2016, 17, 704-714. [CrossRef] [PubMed]

79. Wong, J.J.; Au, A.Y.; Ritchie, W.; Rasko, J.E. Intron retention in mRNA: No longer nonsense: Known and putative roles of intron retention in normal and disease biology. Bioessays 2016, 38, 41-49. [CrossRef] [PubMed]

80. Sznajder, Ł.J.; Thomas, J.D.; Carrell, E.M.; Reid, T.; McFarland, K.N.; Cleary, J.D.; Oliveira, R.; Nutter, C.A.; Bhatt, K.; Sobczak, K.; et al. Intron retention induced by microsatellite expansions as a disease biomarker. Proc. Natl. Acad. Sci. USA 2018, 115, 4234. [CrossRef] [PubMed]

81. Matoulkova, E.; Michalova, E.; Vojtesek, B.; Hrstka, R. The role of the 3' untranslated region in post-transcriptional regulation of protein expression in mammalian cells. RNA Biol. 2012, 9, 563-576. [CrossRef] [PubMed]

82. Hinnebusch, A.G.; Ivanov, I.P.; Sonenberg, N. Translational control by 5'-untranslated regions of eukaryotic mRNAs. Science 2016, 352, 1413-1416. [CrossRef] [PubMed]

(C) 2018 by the authors. Licensee MDPI, Basel, Switzerland. This article is an open access article distributed under the terms and conditions of the Creative Commons Attribution (CC BY) license (http:/ / creativecommons.org/licenses/by/4.0/). 